\section{BMJ Open} Ophthalmology

\title{
Selective laser trabeculoplasty (SLT) performed by optometrists for patients with glaucoma and ocular hypertension: a scoping review
}

\author{
Lee Jones (D) , ${ }^{1,2}$ Evgenia Konstantakopoulou, ${ }^{1,2,3}$ Gus Gazzard (D) 1,2
}

\begin{abstract}
To cite: Jones L, Konstantakopoulou E, Gazzard G. Selective laser trabeculoplasty (SLT) performed by optometrists for patients with glaucoma and ocular hypertension: a scoping review. BMJ Open Ophthalmology 2021;6:e000611. doi:10.1136/ bmjophth-2020-000611
\end{abstract}

- Additional material is published online only. To view, please visit the journal online (http://dx.doi.org/10.1136/ bmjophth-2020-000611).

Received 26 August 2020 Revised 16 January 2021 Accepted 6 February 2021

Check for updates

(C) Author(s) (or their employer(s)) 2021. Re-use permitted under CC BY-NC. No commercial re-use. See rights and permissions. Published by BMJ.

${ }^{1}$ Moorfields Eye Hospital NHS Foundation Trust, London, UK ${ }^{2}$ Institute of Ophthalmology, University College London, London, UK

${ }^{3}$ Division of Optics and Optometry, University of West Attica, Attica, Greece

Correspondence to Professor Gus Gazzard; a. gazzard@ucl.ac.uk

\section{ABSTRACT}

Selective laser trabeculoplasty (SLT) has been established as an effective treatment to lower intraocular pressure in people with glaucoma and ocular hypertension. The procedure is typically within the remit of ophthalmologists; however, there is potential to upskill optometrists and other healthcare professionals (HCPs) to deliver the treatment. We conducted a scoping review to identify the current global landscape of HCP-delivered SLT and describe training features, clinical effectiveness and safety. Relevant articles were identified through online database searches and grey literature sources. Four articles were selected for full inclusion. This review identified training programmes for optometrist-delivered SLT in the UK and the USA. The findings indicate that more research is needed to clarify training requirements and clinical effectiveness.

\section{INTRODUCTION}

Glaucoma comprises a group of diseases characterised by structural damage of the optic nerve and progressive visual field loss. The condition is strongly associated with elevated intraocular pressure (IOP), but also occurs when IOP is within the normal expected range. ${ }^{1}$ Raised IOP without the presence of optic neuropathy is known as ocular hypertension (OHT). The treatment goal in both conditions is to preserve vision, usually through sustained lowering of IOP.

Patients with both OHT and glaucoma require lifelong monitoring, usually within the hospital eye service (HES). However, a rising patient caseload presents service delivery challenges. For example, it is projected that over 6 million people in Europe will be diagnosed with primary open-angle glaucoma by $2040 .^{2}$ Moreover, an increasing shortfall in the number of ophthalmologists worldwide signals that the ophthalmic workforce may struggle to accommodate demand. ${ }^{34}$ This is a significant problem, as delays in treatment initiation, escalation or monitoring may cause considerable harm among patients with glaucoma,${ }^{56}$ which may be detrimental to vision and quality of life. ${ }^{7}$ To address such problems, in $2018,86 \%$ of HES units in the UK relied on non-medical professionals working in extended roles to provide service to some extent. ${ }^{4}$

Optometrists and other healthcare professionals (HCPs) are increasingly supporting the demand profile faced by the HES. For example, many optometrists, orthoptists and nurses have extended their scope of practice in the glaucoma sector. ${ }^{8}$ Studies have consistently demonstrated equivalence in glaucoma clinical decision-making between specially trained optometrists and ophthalmologists. ${ }^{9-12}$ Given the ongoing capacity restraints, HCPs' clinical role will likely continue to develop.

The findings of the Laser in Glaucoma and Ocular Hypertension (LiGHT) trial recently indicated that selective laser trabeculoplasty (SLT) is a safe and effective treatment for people with glaucoma and OHT. ${ }^{13}$ Following the outcomes of the LiGHT trial, the National Institute for Health and Care Excellence is updating guidance to recognise the role of SLT as a first-line treatment option for patients with newly diagnosed glaucoma or OHT. ${ }^{14}$ Between 2019 and 2020, there were approximately 11000 SLT attendances in the UK HES, which is the highest number of annual attendances in the last 10 years and a $33 \%$ increase from the previous year. ${ }^{15}$ The treatment is typically delivered by an ophthalmologist, thus potentially extending resource demand issues. Upskilling non-medical professionals to support delivery of SLT has already emerged in a small number of units within the HES. Specifically, optometrists are good candidates to enable wider usage of SLT due to their proficiency in necessary clinical skills such as gonioscopy and in managing patients with glaucoma. Similarly, ophthalmic nurses have been performing ophthalmic lasers such as yttrium aluminium garnet 
(YAG) capsulotomies and iridotomies. Yet, an evidence synthesis of the available literature surrounding HCPdelivered SLT has not been performed. This research gap is significant, as understanding the various infrastructure currently in place will guide clinical decision-makers when implementing this model of healthcare delivery and may also help identify further research priorities.

\section{Objectives}

- Identify the current infrastructure of SLT delivery by HCPs in the UK and worldwide.

- Describe the training procedures for this model of healthcare delivery.

- Collect and summarise evidence on clinical effectiveness, safety and cost-effectiveness of existing infrastructures where SLT is delivered by HCPs.

\section{METHODS}

This scoping review was conducted using the Preferred Reporting Items for Systematic Reviews and MetaAnalyses (PRISMA) extension for Scoping Reviews checklist ${ }^{16}$ following our published protocol. ${ }^{17}$

\section{Eligibility criteria}

Research studies of any design and methodological approach were included, with no limit on publication dates. Only studies where the full text was available and in English language were included. Eligible studies were required to address the exposure of interest (ie, glaucoma or OHT). There was no requirement for studies to include specific outcomes relating to, for example, efficacy of HCP-delivered SLT. While a broad range of search terms relating to non-medical professionals were included, optometrists are the main focus of this scoping review, due to the lack of records focusing on other specialties.

We excluded articles where the aim was to measure equivalence of SLT outcomes between ophthalmologists in training and consultant ophthalmologists, as the purpose of this review was to investigate non-medical SLT training and delivery.

\section{Search strategy}

The search strategy is described in detail in the review protocol, ${ }^{17}$ and we briefly summarise here. The search was conducted in the following databases: Complete Cumulative Index of Nursing and Allied Health Literature (CINAHL), via EBSCOhost; MEDLINE Complete; Embase; Health Management Information Consortium (HMIC) and Ovid Emcare, via Ovid. All databases were searched from inception date. We used Medical Subject Headings to develop the search terms.

To accompany our database search, we conducted a search of relevant grey literature sources (ie, clinical guidelines and reports). The grey literature search strategy was decided among the research team with consultation from the National Institute for Health Research Applied Research Collaboration North Thames. The online resources for the grey literature search were professional societies worldwide, where non-medical professionals represent the primary ophthalmic healthcare force (ie, USA, Canada, Australia, New Zealand, South Africa and the UK); national health departments; medicine regulatory bodies; charities, professional magazines and conference proceedings. We also conducted a canvassing exercise whereby professional societies in different global regions were contacted by email to enquire about current status of HCP-delivered SLT. To the authors' knowledge, optometrists are the main primary ophthalmic caregiver worldwide, following ophthalmologists. A standardised email enquiring about current jurisdiction on optometrist-delivered SLT was sent to the Canadian Association of Optometrists, American Optometric Association, Optometry Australia, New Zealand Association of Optometrists and the South African Optometric Association. These bodies were selected based on the judgement of the research team and because the expanding practice of optometrists would likely be most relevant within these countries. It was established that delivery of SLT by optometrists is currently prohibited in Canada, Australia and New Zealand, whereas the situation in South Africa could not be established.

\section{Search terms}

('ophthalmologist*' OR 'hospital medical staff*' OR 'junior physician*' OR 'hospital registrar*' OR 'hospital attending physician*' OR 'hospital medical staff*' OR 'resident*' OR 'nonmedical internship*' OR 'nonmedical residenc*' OR 'non-medical internship*' OR 'non-medical residenc*' OR 'allied health personnel' OR 'allied health professional*' OR 'healthcare assistant*' OR 'healthcare support worker*' OR 'paramedic*' OR 'paramedic personnel' OR 'population programme specialist*' OR 'optometrist*') AND ('trabeculoplast*' OR 'trabeculectom*' OR 'goniotom*').

\section{Study selection, data charting and synthesis}

Two reviewers (LJ and EK) independently screened the titles and abstracts of the identified studies using the inclusion/exclusion criteria that were decided a priori. The studies were assessed for suitability using a webbased screening software (Covidence; Veritas Health Innovation, Melbourne, Australia; available at http:// www.covidence.org). Data were charted following recommended methods, ${ }^{16}$ whereby a custom form was developed in Microsoft Excel to capture relevant details within the articles and guidelines. The full list of data items is provided as online supplemental table 1. Following recommendations from the Cochrane Collaboration, we contacted the authors of eligible primary studies via email to obtain additional information on unclear items. We used a narrative synthesis whereby the relevant literature was used to generate a comprehensive evidence base of the infrastructure of SLT delivered by non-medical professionals. The study findings were grouped depending on the similarities and differences in the outcomes assessed. 


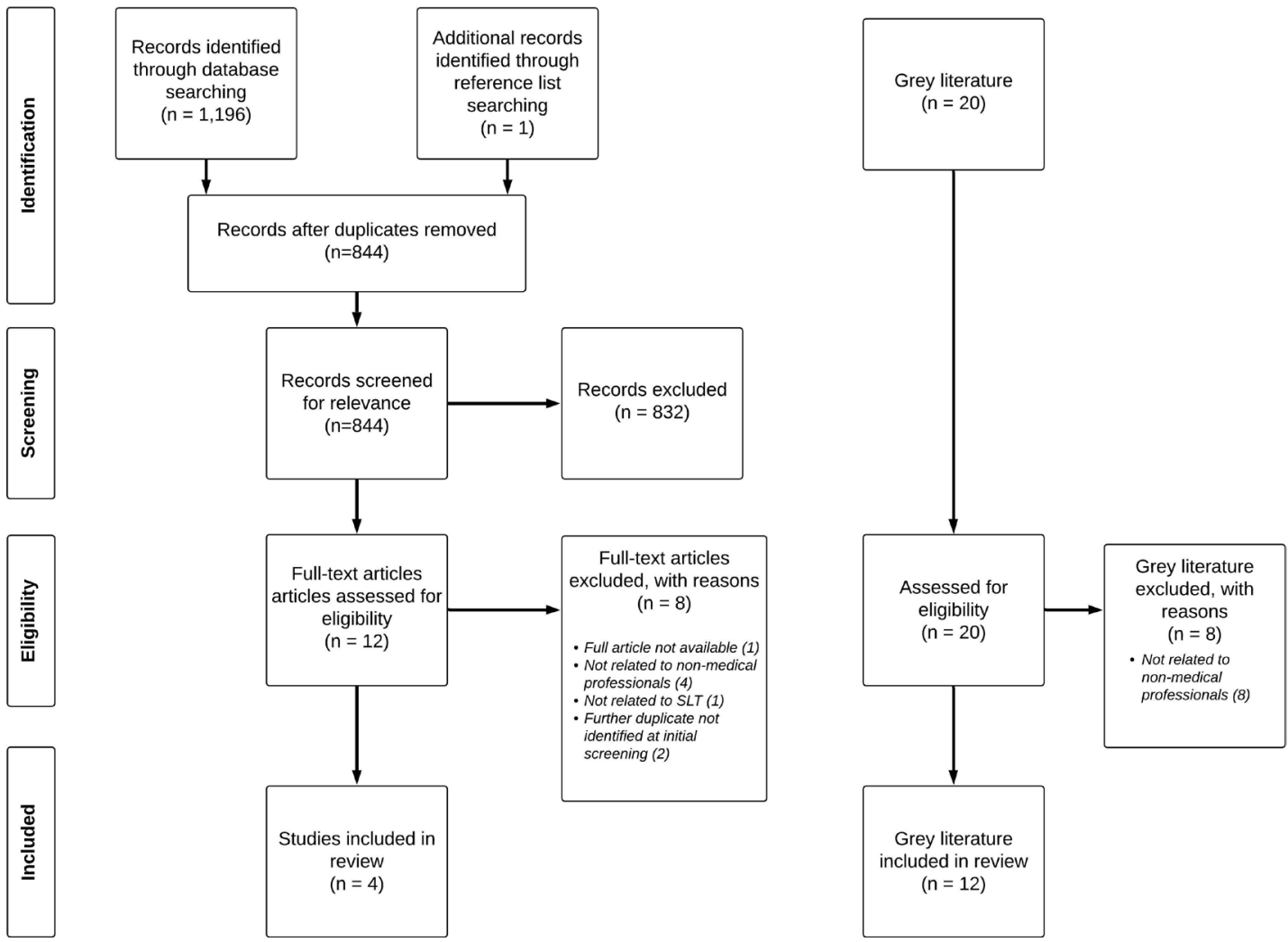

Figure 1 Preferred Reporting Items for Systematic Reviews and Meta-Analyses (PRISMA) diagram showing study selection process. SLT, selective laser trabeculoplasty.

\section{RESULTS}

Searches were run on 6 December 2019 and yielded 1196 records. Sources identified per database were 909 via Ovid and 287 via EBSCOhost. One additional record was identified through backward reference searching. Of these, 353 were automatically removed as duplicates. This left 844 studies to screen using title and abstract, of which 832 were excluded and 12 articles were assessed for fulltext eligibility. A further eight studies were excluded following full-text review, mainly because they were not related to non-medical professionals. Ultimately, four full-text articles were selected for inclusion. For full details of included articles, see the data extraction table (online supplemental table 2). It was decided that an assessment of research quality would be meaningful to help orientate readers given the few number of studies in this area. Studies were assessed for quality using Kmet et al's Standard Quality Assessment Criteria for Evaluating Primary Research Papers from a Variety of Fields. ${ }^{18}$ The assessment criteria and outcomes of the quality appraisal are provided in online supplemental table 3 . One paper could not be quality assessed as it was an invited commentary. Differences in scores were negligible (range: $0.80-1.00$ ) indicating equivalence in the quality of the included studies' methodology and reporting. We identified 12 sources of relevant grey literature. The study selection process is shown in the PRISMA diagram in figure 1. The studies identified presented data predominantly on optometrist-delivered SLT, with one study also including one orthoptist.

\section{DISCUSSION}

\section{Training}

Two discrete training models were described in the literature. One model was based in the USA where optometrists can undertake a course designed to train multiple individuals simultaneously. ${ }^{19}{ }^{20}$ A second model, based in the UK, described a work-based skills matrix to be integrated into practising non-medical professionals' training and development. ${ }^{21}$ Variations in undergraduate training (online supplemental table 4) as well as the type, structure and duration of SLT training delivery make direct comparisons difficult. We summarise the key elements of both SLT training programmes here.

\section{US SLT training programme for optometrists}

At the time of this review, six of the 50 US states (12\%) had passed legislation to allow optometrists to perform laser procedures, namely Oklahoma, Kentucky, Louisiana, Indiana, Alaska and Arkansas. ${ }^{2022-25}$ Our search resulted in information from one single centre in the USA. ${ }^{1920}$ The continuing education programme in the USA is delivered by three optometrists and one ophthalmologist through a 2-day course and has four components. Lectures are delivered on laser physics and tissue interactions, indications and contraindications, alternative treatment options, risks and benefits, potential complications and 
procedural techniques through video-based learning. Laboratory-based practical sessions using model eyes are used for SLT and other forms of laser, with a station on gonioscopy also included. ${ }^{19}{ }^{20}$ Further training components include an assessment of both theory and practical elements of training. Lectures and laboratory sessions are delivered over 10 and 6 hours, respectively. The course is approved by the Council on Optometric Practitioner Education, which is the nationally recognised accreditation programme for optometric continuing education in the USA. Currently, 23 of the 24 schools or colleges of optometry in the USA and Canada have embedded within their curriculum the above laser training.

\section{UK work-based SLT skills matrix for optometrists and orthoptists}

In the UK, there is no university training on the use of SLT or other ophthalmic laser. Chadwick et al report training of non-medical professionals, specifically optometrists and orthoptists, in SLT in Scotland. ${ }^{21}$ The scheme described in this study used a bespoke accreditation framework requiring attendance of a laser safety training lecture and supervised practice under the supervision of a consultant ophthalmologist specialising in glaucoma. This was delivered at a local level within the HES. The training protocol comprised: attendance of a laser induction lecture delivered by a consultant ophthalmologist; conversance with safety guidelines, laser system and operator manual; completion of at least five SLT procedures to the satisfaction of a medical supervisor; a written examination; and completion of a skills checklist of demonstrable abilities such as equipment set-up, understanding of consenting policy and effective communication of the procedure and follow-up care. Chadwick et al reported HCPs performed up to 20 SLT procedures over the course of up to 6 months before being sufficiently confident in the technique, suggesting a possible need to nurture self-confidence as opposed to acquiring competence. ${ }^{21}$ Attainment of independent prescribing (IP) capabilities was a requirement for optometrists, and an equivalent degree of experience was required for orthoptists. Successful training was achieved following competency approval by a consultant ophthalmologist, with a requirement for annual reaccreditation.

Moorfields Eye Hospital (MEH) in the UK has also produced a protocol for training optometrists in SLT. Optometrists attend a laser induction lecture and have to be conversant with the MEH laser safety guideline and the available laser system and operator manual. A total of five SLT procedures are performed by the optometrist under the supervision of a consultant ophthalmologist, who signs off the optometrist once a satisfactory level of competence has been established. There are no clinical experience prerequisites specified in the protocol; the candidacy and selection of optometrists is, however, done following recommendations by consultant ophthalmologists and refers only to optometrists having experience in consultant-led glaucoma clinics. The service is subject to an annual audit and the protocol is reviewed every 3 years.

\section{Postgraduate qualifications}

The skills matrix described by Chadwick et al suggested optometrists were required to achieve IP status, whereas orthoptists were required to have equivalent experience as judged by a consultant ophthalmologist, ${ }^{21}$ the criteria for which were not defined. Medication is routinely prescribed after SLT, mainly to treat spikes in IOP. IP enables UK optometrists to prescribe licensed medicines for conditions affecting the eye, removing the need for an ophthalmologist to issue a prescription. In the USA, graduating optometrists, in all but one state, are licensed to prescribe topical medications for glaucoma. Chadwick et al speculated that IP may not be an essential prerequisite for training in SLT and acknowledge that wider implementation of this model could be restricted if IP qualification is required. Qualitative work is already underway to investigate the necessary postgraduate training expected by optometrists and other HCPs in the UK. ${ }^{17}$

\section{Clinical effectiveness}

Two studies have examined clinical effectiveness of HCPdelivered SLT. For the purposes of this review, clinical effectiveness was defined as successful reduction of IOP and requirement of subsequent SLT session.

Chadwick et al conducted a prospective analysis of outcomes of 207 SLT procedures performed by three nonmedical professionals (two optometrists; one orthoptist) which were compared with eyes treated by an ophthalmologist. ${ }^{21}$ Mean IOP at baseline for the non-medical professional group was $20.9 \pm 5.1 \mathrm{~mm} \mathrm{Hg}$, reducing to $17.0 \pm 4.4 \mathrm{~mm} \mathrm{Hg}$ at 1-month follow-up, demonstrating a median IOP reduction of $16.0 \%$ (IQR: $5.9 \%-28.0 \%$ ). Median IOP reduction of $21.1 \%(9.8 \%-31.4 \%)$ and $17.4 \%$ $(5.6 \%-25.0 \%)$ was reported at 9 and 12 months, respectively. No differences were observed when comparing IOP reduction at 12 months between non-medical professional-treated eyes (mean $\mathrm{IOP}=16.8 \pm 4.3 \mathrm{~mm}$ $\mathrm{Hg} ; \mathrm{n}=21$ ) and ophthalmologist-treated eyes (mean $\mathrm{IOP}=19.0 \pm 5.5 \mathrm{~mm} \mathrm{Hg} ; \mathrm{n}=73$ ) suggesting clinical effectiveness of SLT was equivalent between groups. Limitations of the study were that patients were not randomised between groups, and patients in the ophthalmologisttreated series had statistically significantly higher baseline IOP than those treated by non-medical professionals. As such, ascertainment of clinical effectiveness between the groups is challenging. As described elsewhere, ${ }^{26}$ few studies have used a pragmatic approach to determine SLT clinical effectiveness, such as inclusion of individualised target IOPs and assessing pursuit of control. This method would allow for control over patient factors such as baseline IOP and provide a robust estimate of clinical effectiveness.

A retrospective study comparing outcomes of laser trabeculoplasty performed by optometrists and ophthalmologists 
was conducted by Stein and colleagues using Medicare healthcare insurance data in the USA. ${ }^{27}$ A total of 1150 eyes receiving trabeculoplasty from 57 ophthalmologists were compared with 234 eyes treated by 23 optometrists. Over the 72-month follow-up, repeat trabeculoplasty was required in $84(35.9 \%)$ and $174(15.1 \%)$ of eyes treated by an optometrist and ophthalmologist, respectively. Repeat procedure within 6 months of initial treatment was required for 3.9\% of ophthalmologist-treated eyes compared with $24.9 \%$ of optometrist-treated eyes $(\mathrm{p}<0.001)$. A number of limitations have been identified raising questions over the suitability of the comparisons made. Fingeret noted that at the time of the study the optometrists were (by training) advised to treat $180^{\circ}$ of the trabecular meshwork and to consider treating the remaining $180^{\circ}$ if IOP was not sufficiently stabilised. ${ }^{28}$ As such, repeat trabeculoplasty may indicate clinical guideline differences between professional groups, or a more cautionary approach by optometrists. ${ }^{29}$ Data relating to SLT outcomes were unavailable and procedure utilisation rates were used as a surrogate for clinical effectiveness. The suitability of estimating clinic effectiveness using this method can be questioned due to missingness of clinical information. In addition, the analysis did not control for variances in clinical technique, for example, number of shots and energy level.

Clinical effectiveness of SLT will also be predicted by patient factors and treatment parameters. For example, recent analysis from the LiGHT trial demonstrated that total SLT power and 2-month IOP were predictors of drop-free disease control at 36 months after single SLT. ${ }^{30}$ Moreover, patients who are more medically complex tend to be treated by an ophthalmologist as opposed to an optometrist which should be controlled for when making comparisons between group outcomes. ${ }^{31}$

\section{Safety}

SLT is a low-risk outpatient procedure. Adverse events such as discomfort or mild pain (up to 65.7\%), photophobia (up to $96.7 \%$ ), conjunctival hyperaemia (up to $64 \%$ ) and inflammation of the anterior chamber (up to $89.3 \%$ ) have been reported most commonly in the literature, but are transient and not sight threatening. ${ }^{32}$ IOP spike immediately following SLT has also commonly been reported, although with variable incidence (up to 62\%) and depending on prophylactic IOP-lowering medication (up to $28.8 \%$ ). ${ }^{32}$

Limited data were available regarding the safety of HCPdelivered SLT. Chadwick et al reported minor and/or self-limiting complications in $3.9 \%$ of the eyes treated by two optometrists and one orthoptist, which did not differ significantly from the ophthalmologist-treated eyes $(4.8 \%) .{ }^{21}$ The rate of IOP spike was $0.3 \%$, compared with $1.4 \%$ in the ophthalmologist-delivered SLT series and $0.8 \%$ reported by the LiGHT trial; both of these studies used prophylactic IOP-lowering medication. ${ }^{13}$

\section{Cost-effectiveness}

The included studies did not reveal any evidence on the costeffectiveness of HCP-delivered SLT. An economic evaluation of HCP-delivered SLT would be essential before the implementation of any service changes, as extended roles and increased responsibilities are likely to be reflected in higher pay scales for HCPs.

\section{CONCLUSIONS}

Implementation of SLT clinics led by optometrists and/or other HCPs may help strengthen the overall glaucoma care service by increasing opportunity to promptly treat people with glaucoma and OHT, while allowing ophthalmologists to prioritise more complex cases. The extent of optometrists and other HCPs' clinical readiness to deliver SLT will be affected by previous training; global differences in teaching approaches may partly explain the inconsistent findings observed in this review.

Our review highlights the limited number of studies that have assessed clinical effectiveness of HCP-delivered SLT. Although limited, published results indicate HCP outcomes are comparable to ophthalmologists. The level of evidence was low as outcomes have only been assessed among a small number of HCPs without optimising study design. There has yet to be a randomised controlled clinical trial evaluating outcomes, such as clinical efficacy, safety profile, legal framework and patient experience in HCP-delivered SLT. Further research into stakeholders' views of changes in the delivery infrastructure of SLT (already underway by the review authors) and into clinical effectiveness and costeffectiveness and safety, with suitable outcome measures and larger sample sizes, may help consolidate evidence in this area.

Contributors All authors made substantial contributions to the design and analysis of the work. LJ performed the literature search and manuscript preparation. EK conceptualised the review and performed the literature search. GG conceptualised the review. All authors approved the final manuscript.

Funding This study was funded by Moorfields Eye Charity (GR001067).

Competing interests None declared.

Patient consent for publication Not required.

Provenance and peer review Not commissioned; externally peer reviewed. Data availability statement No data are available.

Supplemental material This content has been supplied by the author(s). It has not been vetted by BMJ Publishing Group Limited (BMJ) and may not have been peer-reviewed. Any opinions or recommendations discussed are solely those of the author(s) and are not endorsed by BMJ. BMJ disclaims all liability and responsibility arising from any reliance placed on the content. Where the content includes any translated material, BMJ does not warrant the accuracy and reliability of the translations (including but not limited to local regulations, clinical guidelines, terminology, drug names and drug dosages), and is not responsible for any error and/or omissions arising from translation and adaptation or otherwise.

Open access This is an open access article distributed in accordance with the Creative Commons Attribution Non Commercial (CC BY-NC 4.0) license, which permits others to distribute, remix, adapt, build upon this work noncommercially, and license their derivative works on different terms, provided the original work is properly cited, appropriate credit is given, any changes made indicated, and the use is non-commercial. See: http://creativecommons.org/ licenses/by-nc/4.0/.

\section{ORCID iDs}

Lee Jones http://orcid.org/0000-0002-8030-1211

Gus Gazzard http://orcid.org/0000-0003-1982-5005 


\section{REFERENCES}

1 Chan MPY, Broadway DC, Khawaja AP, et al. Glaucoma and intraocular pressure in EPIC-Norfolk eye study: cross sectional study. BMJ 2017;358:j3889.

2 Tham Y-C, Li X, Wong TY, et al. Global prevalence of glaucoma and projections of glaucoma burden through 2040: a systematic review and meta-analysis. Ophthalmology 2014;121:2081-90.

3 Resnikoff S, Felch W, Gauthier T-M, et al. The number of ophthalmologists in practice and training worldwide: a growing gap despite more than 200,000 practitioners. Br J Ophthalmol 2012;96:783-7.

4 The Royal College of Ophthalmologists. Workforce census, 2018. Available: https://www.rcophth.ac.uk/wp-content/uploads/2019/02/ RCOphth-Workforce-Census-2018.pdf [Accessed 04 Aug 2020].

5 Tatham A, Murdoch I. The effect of appointment rescheduling on monitoring interval and patient attendance in the glaucoma outpatient clinic. Eye 2012;26:729-33.

6 Healthcare Safety Investigation Branch. Lack of timely monitoring of patients with glaucoma, 2020. Available: https://www.hsib.org. uk/documents/199/hsib_report_lack_timely_monitoring_patients_ glaucoma.pdf [Accessed 04 Aug 2020].

7 Jones L, Bryan SR, Crabb DP. Gradually then suddenly? decline in vision-related quality of life as glaucoma worsens. J Ophthalmol 2017;2017:1-7.

8 Harper R, Creer R, Jackson J, et al. Scope of practice of optometrists working in the UK Hospital eye service: a national survey. Ophthalmic Physiol Opt 2016;36:197-206.

9 Banes MJ, Culham LE, Bunce C, et al. Agreement between optometrists and ophthalmologists on clinical management decisions for patients with glaucoma. $\mathrm{Br} J$ Ophthalmol 2006;90:579-85.

10 Azuara-Blanco A, Burr J, Thomas R, et al. The accuracy of accredited glaucoma optometrists in the diagnosis and treatment recommendation for glaucoma. Br J Ophthalmol 2007;91:1639-43.

11 Ho S, Vernon SA. Decision making in chronic glaucoma-optometrists vs ophthalmologists in a shared care service. Ophthalmic Physiol Opt 2011;31:168-73.

12 Marks JR, Harding AK, Harper RA, et al. Agreement between specially trained and accredited optometrists and glaucoma specialist consultant ophthalmologists in their management of glaucoma patients. Eye 2012;26:853-61.

13 Gazzard G, Konstantakopoulou E, Garway-Heath D, et al. Selective laser trabeculoplasty versus eye drops for first-line treatment of ocular hypertension and glaucoma (light): a multicentre randomised controlled trial. Lancet 2019;393:1505-16.

14 National institute for health and clinical excellence (NICE). Glaucoma: diagnosis and management. 2019 exceptional surveillance of glaucoma: diagnosis and management (NICE guideline NG81). Available: https://www.nice.org.uk/guidance/ng81/ resources/2019-exceptional-surveillance-of-glaucoma-diagnosisand-management-nice-guideline-ng81-6901877773/chapter/ Surveillance-decision?tab=evidence [Accessed 12 Nov 2020].

15 NHS Digital. Hospital outpatient activity. Available: https://digital.nhs uk/data-and-information/publications/statistical/hospital-outpatientactivity [Accessed 03 Nov 2020].
16 Tricco AC, Lillie E, Zarin W, et al. PRISMA extension for scoping reviews (PRISMA-ScR): checklist and explanation. Ann Intern Med 2018;169:467-73.

17 Jones L, Konstantakopoulou E, Gazzard G. Selective laser trabeculoplasty (SLT) performed by optometrists for patients with glaucoma and ocular hypertension: a scoping review protocol. BMJ Open Ophthalmol 2020;5:e000438.

18 Kmet LM, Lee RC, Cook LS. HTA Initiative \#13. Standard quality assessment criteria for evaluating primary research papers from a variety of fields, 2004. Available: https://www.ihe.ca/advancedsearch/standard-quality-assessment-criteria-for-evaluating-primaryresearch-papers-from-a-variety-of-fields

19 Lighthizer N. Get laser focused on the appropriate glaucoma treatment: SLT-once the exclusive domain of ophthalmology-is becoming a first-line treatment as multiple states allow optometrists to perform it. Rev Optom 2016;153:66-72.

20 Lightizer N. Associate dean, NSU Oklahoma College of Optometry. personal communication, 2020.

21 Chadwick O, Chia SN, Rotchford A. Establishing an allied health professional delivered selective laser trabeculoplasty service in Scotland. Ophthalmic Physiol Opt 2019;39:216-23.

22 Waltz K. From both sides now. The ophthalmologist. Available: https://theophthalmologist.com/business-profession/from-bothsides-now [Accessed 25 Aug 2020].

23 Fingeret M. The Optomestrist's role in glaucoma care. Glaucoma Today. Available: https://glaucomatoday.com/articles/2014-mayjune/the-optometrists-role-in-glaucoma-care [Accessed 25 Aug 2020].

24 American Medical News. Optometrists seek surgery rights in more states after Kentucky victory. Available: https://amednews.com/ article/20110523/profession/305239946/2/ [Accessed 25 Aug 2020].

25 Ophthalmology Times. Kentucky bill expands laser scope for optometrists. Available: https://www.ophthalmologytimes.com/view/ kentucky-bill-expands-laser-scope-optometrists.[Accessed 25 Aug 2020]

26 Garg A, Gazzard G. Selective laser trabeculoplasty: past, present, and future. Eye 2018;32:863-76.

27 Stein JD, Zhao PY, Andrews C, et al. Comparison of outcomes of laser trabeculoplasty performed by optometrists vs ophthalmologists in Oklahoma. JAMA Ophthalmol 2016;134:1095-101.

28 Fingeret M. Laser trabeculoplasty use patterns among optometrists and ophthalmologists in Oklahoma. JAMA Ophthalmol 2016;134:1101-2.

29 Robin AL. Public health policy lessons from Oklahoma. JAMA Ophthalmol 2016;134:1102-3.

30 Garg A, Vickerstaff V, Nathwani N, et al. Primary selective laser trabeculoplasty for open-angle glaucoma and ocular hypertension: clinical outcomes, predictors of success, and safety from the laser in glaucoma and ocular hypertension trial. Ophthalmology 2019;126:1238-48.

31 Miller DD, Stewart MW, Gagne JJ, et al. Differences in characteristics of Medicare patients treated by ophthalmologists and optometrists. PLoS One 2020;15:2019-12.

32 Wong MOM, Lee JWY, Choy BNK, et al. Systematic review and meta-analysis on the efficacy of selective laser trabeculoplasty in open-angle glaucoma. Surv Ophthalmol 2015;60:36-50. 